\title{
APPROXIMATION BY GENERALIZED IMPEDANCE BOUNDARY CONDITIONS OF A TRANSMISSION PROBLEM IN ACOUSTIC SCATTERING
}

\author{
XAvier Antoine ${ }^{1}$ And HÉLÈne BARUCQ ${ }^{2}$
}

\begin{abstract}
This paper addresses some results on the development of an approximate method for computing the acoustic field scattered by a three-dimensional penetrable object immersed into an incompressible fluid. The basic idea of the method consists in using on-surface differential operators that locally reproduce the interior propagation phenomenon. This approach leads to integral equation formulations with a reduced computational cost compared to standard integral formulations coupling both the transmitted and scattered waves. Theoretical aspects of the problem and numerical experiments are reported to analyze the efficiency of the method and precise its validity domain.
\end{abstract}

Mathematics Subject Classification. 35J05, 35J25, 35S15, 65N38, 78A45.

Received: February 25, 2004. Revised: March 7, 2005.

\section{INTRODUCTION}

Since the advent of the fast multipole method (FMM) [30], the technique of integral equations [16] has became one of the most efficient and useful methods in computational acoustics for solving the Helmholtz equation. Its principle consists in rewriting the initial problem as an equation set onto the surface of the considered object and leads to a gain of one dimension space. This equation is integro-differential and therefore non-local. If one considers the practical point of view of their numerical approximation by a boundary element method, the number of degrees of freedom $n_{\lambda}$ of the dense complex and generally non-Hermitian linear system can be of several millions when the wavelength $\lambda$ of the incident signal is small compared to the size of the target. Instead of a direct Gauss elimination solver, the linear system is nowadays often solved by a Krylov subspace iterative solver (GMRES, CGS, QMR, etc.) [32]. Consequently, the total cost of a such algorithm to get a sufficiently accurate solution is $\mathcal{O}\left(n_{\varepsilon}^{i t e r} n_{\lambda}^{2}\right)$, where $\mathcal{O}\left(n_{\lambda}^{2}\right)$ comes from the Matrix-Vector (MV) products involved at each step of the iterative solver and $n_{\varepsilon}^{i t e r}$ designates the number of iterations required to compute the solution with a tolerance $\varepsilon$. The quadratic cost of a MV evaluation can actually be reduced to $\mathcal{O}\left(n_{\lambda} \log n_{\lambda}\right)$ for instance by the FMM $[19-21,30]$. The number of iterations $n_{\varepsilon}^{\text {iter }}$ can be diminished by using a suitable preconditioning strategy of the linear system $[10,11,13]$ or the integral equation $[6,7,14,26,27,38]$.

Keywords and phrases. Helmholtz equation, acoustics, integral equations, generalized impedance boundary conditions, existence and uniqueness results.

${ }^{1}$ Université Paul Sabatier, Laboratoire de Mathématiques pour l'Industrie et la Physique (CNRS UMR 5640), UFR MIG, 118, route de Narbonne, 31062 Toulouse Cedex 4, France. antoine@mip.ups-tlse.fr

${ }^{2}$ Université de Pau et des Pays de l'Adour, Laboratoire de Mathématiques Appliquées (CNRS FRE 2570), IPRA, avenue de l'Université, 64000 Pau, France.

(c) EDP Sciences, SMAI 2005 
Upstream from the problem of solving the linear system by a preconditioned Krylov/FMM solver is posed the question of the construction of an integral equation which gives an accurate computation of the scattered field and minimizes the size $n_{\lambda}$ of the system. The present work is placed in this framework. More precisely, the problem under consideration here is the scattering of a time-harmonic acoustic wave by a three-dimensional bounded object $\Omega_{1}$ immersed into an incompressible fluid occupying the complementary domain $\Omega_{2}=\mathbb{R}^{3} / \overline{\Omega_{1}}$. The regular and arbitrarily shaped interface $\Gamma$ between the two media is here supposed to coincide with the boundary of $\Omega_{1}$. The total field associated to the diffraction phenomenon is given as the solution to a transmission problem coupling two Helmholtz equations in each medium for two distinct wavenumbers $k_{1}$ in $\Omega_{1}$ and $k_{2}$ in $\Omega_{2}$. To numerically solve such a problem, one may solve a system of coupled equivalent integral equations $[8,16]$ set on $\Gamma$. This provides an accurate solution $[9,18,24]$. However, the handling of two distinct domains defined by some different constitutive parameters involves two wavelengths: $\lambda_{1}$ and $\lambda_{2}$. The number of degrees of freedom is then fixed by the so-called "rule of the thumb" which corresponds to taking a certain number of points per wavelength. Therefore if $\left|k_{1}\right| \gg\left|k_{2}\right|$, the size of the mesh is linked to the smallest wavelength $\lambda_{1}$. Moreover, this approach has also the drawback (see Sect. 2) of working with two surfacic densities: the two first traces $(p, \zeta)$ of the acoustic field at the interface. If we proceed to a boundary element approximation, the size $n^{e x}$ of the resulting linear system for this exact formulation is $3 N V_{\lambda_{1}}$, where $N V_{\lambda_{1}}$ designates the number of vertices of the triangular mesh discretized with respect to the smallest wavelength $\lambda_{1}$.

A possible solution to work with only one surface field and to deal with a size $n^{a p p}=N V_{\lambda_{2}}$ which only depends on the exterior wavelength $\lambda_{2}$ (whence reducing drastically the size of the linear system since $N V_{\lambda_{2}} \ll N V_{\lambda_{1}}$ ) consists in replacing the exact interior integral equation by a localized approximate one proceeding to an asymptotic analysis in the high frequency regime assuming $\left|k_{1}\right| \gg\left|k_{2}\right|$. This kind of equation is usually called a generalized impedance boundary condition [36] and can be written as

$$
\partial_{\mathbf{n}} w=Y w, \text { on } \Gamma
$$

where $Y$ is a differential operator including both the constitutive physical parameters and the geometrical characteristics of the scatterer $\Omega_{1}$. The simplest condition is the so-called Fourier-Robin boundary condition. It links the normal derivative and the trace of the exterior solution through a complex constant coefficient representing the surface impedance. It seems that the development of higher-order impedance boundary conditions has received less attention in acoustics than in electromagnetism [31,33,34,36,37,41]. In 1992, Jones [23] has investigated an improvement of the On-Surface Radiation Conditions [25] method to construct generalized impedance boundary conditions for the Helmholtz equation in the case of a spherical interface. Next, Senior [35] derived approximate conditions for a flat interface. Recently, we have proposed in [5] a first and a second-order generalized impedance boundary conditions for the three-dimensional acoustic scattering problem by an arbitrarily shaped dissipative body. Using some explicit computations based on Mie series expansions in the case of both the circular cylinder and the spherical scatterer, it has been proved that the second-order condition yields a better accuracy and owns a wider physical and numerical domain of validity than the usual Fourier-Robin condition. The aim of this work is to extend the application range of the second-order generalized impedance boundary condition to a general scatterer by the way of an integral equation framework. It can be noticed that other kinds of numerical procedures may also be considered by using for instance a three-dimensional finite element method coupled to an efficient iterative solver and a non-reflecting boundary condition [3,22].

The plan of the paper is the following. In Section 2, we fix the notations relative to the transmission acoustic scattering boundary-value problem. Next, we recall the derivation of the system of equivalent integral equations to solve and its boundary element approximation following [9]. In the third section, we introduce the approach based on the first and second-order generalized impedance boundary conditions derived in [5]. We state some results on existence and uniqueness of the solution to the generalized impedance boundary-value problem under some physical and geometrical sufficient conditions. Next, we proceed to a numerical study of the validity domain of these two impedance conditions for a spherical scatterer. It appears that the numerical domain of application exactly coincides with the theoretical one formerly drawn to ensure the well-posedness. In a fourth section, we briefly describe the integral equation framework for the second-order generalized impedance boundary condition 
and focus on some aspects of its numerical implementation using boundary elements. This provides a numerical procedure with a cost close to the one usually required for the solution of a Neumann boundary-value problem in the exterior computational domain. Finally, we analyze the accuracy and computational cost of the exact and approximate integral equations to show the efficiency and sometimes also certain limitations of the proposed approach.

\section{THE TRANSMISSION PROBLEM}

\subsection{Notations and problem setting}

Let us consider a bounded domain $\Omega_{1} \subset \mathbb{R}^{3}$ whose boundary $\Gamma$ is $\mathcal{C}^{\infty}$ and the associated domain of propagation $\Omega_{2}=\mathbb{R}^{3} \backslash \overline{\Omega_{1}}$. We suppose that each medium $\Omega_{j}, j=1,2$, is homogeneous and isotropic. As a consequence, the density and sound velocity are two positive real constants respectively denoted by $\rho_{j}$ and $c_{j}$ for each domain $\Omega_{j}$. In the sequel, we make the assumption that $\Omega_{1}$ is a dissipative medium characterized by a real strictly positive damping coefficient $\delta$.

Let $u_{0}$ be a complex-valued time-harmonic incident field defined in an open neighborhood $\mathcal{V}$ of $\Gamma$ and satisfying the Helmholtz equation

$$
\Delta u_{0}+k_{2}^{2} u_{0}=0, \quad \text { in } \mathcal{V} .
$$

Hereabove, $k_{2}$ designates the wavenumber in the exterior domain. Such an equation results from removing the sinusoidal time dependence $\mathrm{e}^{-i \omega t}$ in the linear wave equation. The wavenumber $k_{2}$ is related to the pulsation $\omega$ by $k_{2}=\omega / c_{2}$. A second wavenumber $k_{1}$ relative to the interior dissipative domain is defined as

$$
k_{1}^{2}=\frac{\omega^{2}}{c_{1}^{2}}\left(1+i \frac{\delta}{\omega}\right)
$$

A quite usual notation consists in introducing the complex refractive index $N$ and the complex contrast coefficient $\alpha[9]$

$$
N=\frac{1}{c_{r}}\left(1+i \frac{\delta}{\omega}\right)^{1 / 2}, \quad \alpha=\frac{1}{\rho_{r}(1+i \delta / \omega)},
$$

where $c_{r}=c_{1} / c_{2}$ and $\rho_{r}=\rho_{1} / \rho_{2}$ respectively stand for the relative velocity and density. In the sequel, we denote by $z^{1 / 2}$ the principal determination of the square root of $z \in \mathbb{C}$ with branch-cut along the negative real axis. Therefore, the imaginary part of $N$ is strictly positive and $\alpha$ is a complex number with a real part and a non-negative imaginary part. Let us introduce finally $\gamma_{1}=\delta / \omega$.

The scattered field $v$ satisfies the boundary-value problem

$$
\left\{\begin{array}{l}
\Delta v_{2}+k_{2}^{2} v_{2}=0, \quad \text { in } \Omega_{2}, \\
\Delta v_{1}+k_{1}^{2} v_{1}=k_{2}^{2}\left(1-N^{2}\right) u_{0}, \quad \text { in } \Omega_{1}, \\
{[v]=0 \text { and }\left[\chi \partial_{\mathbf{n}} v\right]=-\left[\chi \partial_{\mathbf{n}} u_{0}\right], \quad \text { on } \Gamma,} \\
\lim _{|\mathbf{x}| \rightarrow+\infty}|\mathbf{x}|\left(\nabla v_{2} \cdot \frac{\mathbf{x}}{|\mathbf{x}|}-i k_{2} v_{2}\right)=0
\end{array}\right.
$$

where the piecewise constant function $\chi$ is such that $\chi=1$ in $\Omega_{2}$ and $\chi=\alpha$ in $\Omega_{1}$. Vector $\mathbf{n}$ is the outwardly directed unit normal to $\Omega_{1}$. The restriction of the scattered field $v$ to $\Omega_{j}, j=1,2$, is denoted by $v_{j}:=v_{\mid \Omega_{j}}$; notation [.] designates the jump through $\Gamma$ of a distribution defined in $\Omega_{1} \cup \Omega_{2}$ and is given as the difference between the interior and exterior traces: $[v]:=v_{1 \mid \Gamma}-v_{2 \mid \Gamma}$. If $\mathbf{a}$ and $\mathbf{b}$ are two complex-valued vector fields, their inner product is set as: $\mathbf{a} \cdot \mathbf{b}=\sum_{k=1}^{3} a_{k} \overline{b_{k}}$. Operator $\nabla$ is the gradient operator of a scalar complex field and $\Delta$ designates the Laplace operator. Finally, the last condition in problem (3) is the well-known Sommerfeld radiation condition which ensures the uniqueness of the solution to the boundary-value problem. The notation 
$S R C(v)=0$ means that the given field $v$ satisfies the Sommerfeld radiation condition. We refer to Chazarain and Piriou [12] for any notation concerning the functional spaces (e.g. the Schwartz spaces $\mathcal{D}^{\prime}\left(\Omega_{1}\right)$ or Sobolev spaces $H^{1}\left(\Omega_{1}\right)$ or $\left.H^{1 / 2}(\Gamma)\right)$. Here, $H_{\text {loc }}^{1}\left(\overline{\Omega_{2}}\right)$ is the Sobolev space

$$
H_{\mathrm{loc}}^{1}\left(\overline{\Omega_{2}}\right)=\left\{v \in \mathcal{D}^{\prime}\left(\Omega_{2}\right) / \varphi v \in H^{1}\left(\overline{\Omega_{2}}\right), \forall \varphi \in \mathcal{D}\left(\mathbb{R}^{3}\right)\right\}
$$

Under the above assumptions, we have the following existence and uniqueness result $[4,9]$.

Theorem 2.1. Let $f \in L^{2}\left(\Omega_{1}\right)$ and $g \in H^{-1 / 2}(\Gamma)$. Then, there exists a unique solution $v \in H^{1}\left(\Omega_{1}\right) \cap H_{l o c}^{1}\left(\overline{\Omega_{2}}\right)$ to the transmission boundary-value problem

$$
\left\{\begin{array}{l}
\Delta v+k_{1}^{2} v=f, \text { in } \mathcal{D}^{\prime}\left(\Omega_{1}\right), \\
\Delta v+k_{2}^{2} v=0, \text { in } \mathcal{D}^{\prime}\left(\Omega_{2}\right), \\
{[v]=0, \text { in } H^{1 / 2}(\Gamma),} \\
{\left[\chi \partial_{\mathbf{n}} v\right]=g, \text { in } H^{-1 / 2}(\Gamma),} \\
S R C(v) .
\end{array}\right.
$$

\subsection{Integral equation formulation (IE)}

Let $u=v+u_{0}$ be the total field in $\Omega_{1} \cup \Omega_{2}$. Let us define the Green kernel $G_{j}$ associated to the Helmholtz equation in $\Omega_{j}$ by

$$
G_{j}(\mathbf{x}, \mathbf{y}):=\frac{1}{4 \pi} \frac{\mathrm{e}^{i k_{j}|\mathbf{x}-\mathbf{y}|}}{|\mathbf{x}-\mathbf{y}|} .
$$

We consider the following surface fields $p_{j}:=u_{j \mid \Gamma}$ and $\zeta_{j}:=\chi \partial_{\mathbf{n}} u_{j \mid \Gamma}$, for $j=1,2$. Using the interface conditions arising from (4), the determination of the total field can be reduced to the computation of the quantities: $\zeta:=\zeta_{j}$ and $p:=p_{j}$. Let us consider the following integral operators on $\Gamma$

$$
\begin{aligned}
& V_{j} \zeta(\mathbf{x}):=\int_{\Gamma} G_{j}(\mathbf{x}, \mathbf{y}) \zeta(\mathbf{y}) \mathrm{d} \Gamma(\mathbf{y}), \quad N_{j} p(\mathbf{x}):=\int_{\Gamma} \partial_{\mathbf{n}(\mathbf{y})} G_{j}(\mathbf{x}, \mathbf{y}) p(\mathbf{y}) \mathrm{d} \Gamma(\mathbf{y}) \\
& K_{j} \zeta(\mathbf{x}):=N_{j}^{T} \zeta(\mathbf{x})=\int_{\Gamma} \partial_{\mathbf{n}(\mathbf{x})} G_{j}(\mathbf{x}, \mathbf{y}) \zeta(\mathbf{y}) \mathrm{d} \Gamma(\mathbf{y}), \quad D_{j} p(\mathbf{x}):=\partial_{\mathbf{n}(\mathbf{x})} \int_{\Gamma} \partial_{\mathbf{n}(\mathbf{y})} G_{j}(\mathbf{x}, \mathbf{y}) p(\mathbf{y}) \mathrm{d} \Gamma(\mathbf{y})
\end{aligned}
$$

where $\mathbf{x} \in \Gamma$. The integral operators $V_{j}, N_{j}$ and $K_{j}$ are pseudodifferential operators of order -1 whereas $D_{j}$ is a first-order hypersingular operator (see e.g. [17]).

Let us introduce the product-space $Z=H^{-1 / 2}(\Gamma) \times H^{1 / 2}(\Gamma),\langle.,$.$\rangle the antiduality product between spaces$ $H^{1 / 2}(\Gamma)$ and $H^{-1 / 2}(\Gamma)$ and the integral operators

$$
V:=\alpha^{-1} V_{1}+V_{2}, \quad N:=N_{1}+N_{2}, \quad K:=K_{1}+K_{2}, \quad D:=\alpha D_{1}+D_{2} .
$$

We define the following integral operators

$$
\begin{aligned}
c_{j}(p, q) & :=\iint_{\Gamma \times \Gamma} G_{j}(\mathbf{x}, \mathbf{y}) \operatorname{curl}_{\Gamma} p(\mathbf{y}) \cdot \operatorname{curl}_{\Gamma} \overline{q(\mathbf{x})} \mathrm{d} \Gamma(\mathbf{y}) \mathrm{d} \Gamma(\mathbf{x}), j=1,2, \\
d_{j}(p, q) & :=k_{j}^{2} \iint_{\Gamma \times \Gamma} G_{j}(\mathbf{x}, \mathbf{y}) p(\mathbf{y}) \overline{q(\mathbf{x})} \mathbf{n}(\mathbf{y}) \cdot \mathbf{n}(\mathbf{x}) \mathrm{d} \Gamma(\mathbf{y}) \mathrm{d} \Gamma(\mathbf{x}), j=1,2,
\end{aligned}
$$

and

$$
c(p, q):=\alpha c_{1}(p, q)+c_{2}(p, q), \quad d(p, q):=\alpha d_{1}(p, q)+d_{2}(p, q) .
$$


If $\nabla_{\Gamma}$ designates the surfacic gradient operator, the surfacic curling vector $\operatorname{curl}_{\Gamma}$ is given by $\operatorname{curl}_{\Gamma} \varphi=\nabla_{\Gamma} \varphi \times \mathbf{n}$, for a distribution $\varphi \in \mathcal{D}^{\prime}(\Gamma)$. Let us define the sesquilinear forms

$$
a(\zeta, \mu):=\langle V \zeta, \mu\rangle, \quad b(p, \mu):=\langle N p, \mu\rangle, \quad b^{*}(\zeta, q):=\overline{\langle\bar{V} q, \zeta\rangle}
$$

Basing upon the approach of Bendali and Souilah [9], it can be shown that the solution to the boundary-value problem (4) can be calculated by solving the following system of integral equations:

$$
\left\{\begin{array}{l}
\text { find }(\zeta, p) \in Z \text { solution to } \\
a(\zeta, \mu)-b(p, \mu)=\left\langle u_{0}, \mu\right\rangle, \quad \forall \mu \in H^{-1 / 2}(\Gamma) \\
-b^{*}(\zeta, q)-c(p, q)+d(p, q)=-\overline{\left\langle q, \partial_{\mathbf{n}} u_{0 \mid \Gamma}\right\rangle}, \forall q \in H^{1 / 2}(\Gamma) .
\end{array}\right.
$$

\subsection{The boundary element method}

Let $\Gamma_{h}$ be a polyhedral surface interpolating $\Gamma$ and satisfying the classical non-overlapping condition involved in finite element methods $[9,15]$. Let $\mathcal{T}_{h}=\cup_{i=1}^{N T} K_{i}$ be a triangulation of $\Gamma_{h}$ where each triangle $K_{i}$ satisfies: $K_{i} \cap K_{j}=\emptyset$ for $1 \leq i \neq j \leq N T$. We denote by $\mathcal{P}_{m}$ the space of complex-valued polynomials of degree lower than or equal to $m$. Then, we consider the finite element spaces

$$
M_{h}:=\left\{\zeta \in L^{2}\left(\Gamma_{h}\right) / \zeta_{K}:=\zeta_{\mid K} \in \mathcal{P}_{0}, \forall K \in \mathcal{T}_{h}\right\}, \quad V_{h}:=\left\{q \in \mathcal{C}^{0}\left(\Gamma_{h}\right) / q_{K}:=q_{\mid K} \in \mathcal{P}_{1}, \forall K \in \mathcal{T}_{h}\right\}
$$

We have $\operatorname{Dim} M_{h}=N T$ and $\operatorname{Dim} V_{h}=N V$, where $N V$ denotes the number of vertices arising from the triangulation. If $Z_{h}:=M_{h} \times V_{h}$, the discrete solution $\left(\zeta_{h}, p_{h}\right) \in Z_{h}$ satisfies the formulation (called IE during the numerical experiments at Sect. 4)

$$
\begin{aligned}
& a_{h}\left(\zeta_{h}, \mu_{h}\right)-b_{h}\left(p_{h}, \mu_{h}\right)=\left\langle u_{0 \mid \Gamma_{h}}, \mu_{h}\right\rangle, \forall \mu_{h} \in M_{h}, \\
& -b_{h}^{*}\left(\zeta_{h}, q_{h}\right)-c_{h}\left(p_{h}, q_{h}\right)+d_{h}\left(p_{h}, q_{h}\right)=-\overline{\left\langle q_{h}, \partial_{\mathbf{n}_{h}} u_{0 \mid \Gamma_{h}}\right\rangle}, \forall q_{h} \in V_{h},
\end{aligned}
$$

where the discrete sesquilinear forms are given by: $\forall\left(\zeta_{h}, \mu_{h}\right) \in M_{h} \times M_{h}$ and $\forall\left(p_{h}, q_{h}\right) \in V_{h} \times V_{h}$

$$
\begin{aligned}
a_{h}\left(\zeta_{h}, \mu_{h}\right) & :=\iint_{\Gamma_{h} \times \Gamma_{h}} \sum_{j=1,2} \frac{1}{\chi} G_{j}\left(\mathbf{x}_{h}, \mathbf{y}_{h}\right) \zeta_{h}\left(\mathbf{y}_{h}\right) \overline{\mu_{h}\left(\mathbf{x}_{h}\right)} \mathrm{d} \Gamma_{h}\left(\mathbf{y}_{h}\right) \mathrm{d} \Gamma_{h}\left(\mathbf{x}_{h}\right), \\
b_{h}\left(p_{h}, \mu_{h}\right) & :=\iint_{\Gamma_{h} \times \Gamma_{h}} \sum_{j=1,2} \partial_{\mathbf{n}_{h}\left(\mathbf{y}_{h}\right)} G_{j}\left(\mathbf{x}_{h}, \mathbf{y}_{h}\right) p_{h}\left(\mathbf{y}_{h}\right) \overline{\mu_{h}\left(\mathbf{x}_{h}\right)} \mathrm{d} \Gamma_{h}\left(\mathbf{y}_{h}\right) \mathrm{d} \Gamma_{h}\left(\mathbf{x}_{h}\right), \\
c_{h}\left(p_{h}, q_{h}\right) & :=\iint_{\Gamma_{h} \times \Gamma_{h}} \sum_{j=1,2} \chi G_{j}\left(\mathbf{x}_{h}, \mathbf{y}_{h}\right) \operatorname{curl}_{\Gamma_{h}} p_{h}\left(\mathbf{y}_{h}\right) \cdot \operatorname{curl}_{\Gamma_{h}} \overline{q_{h}\left(\mathbf{x}_{h}\right)} \mathrm{d} \Gamma_{h}\left(\mathbf{y}_{h}\right) \mathrm{d} \Gamma_{h}\left(\mathbf{x}_{h}\right), \\
d_{h}\left(p_{h}, q_{h}\right) & :=\iint_{\Gamma_{h} \times \Gamma_{h}} \sum_{j=1,2} \chi k_{j}^{2} G_{j}\left(\mathbf{x}_{h}, \mathbf{y}_{h}\right) p_{h}\left(\mathbf{y}_{h}\right) \overline{q_{h}\left(\mathbf{x}_{h}\right)} \mathbf{n}_{h}\left(\mathbf{y}_{h}\right) \cdot \mathbf{n}_{h}\left(\mathbf{x}_{h}\right) \mathrm{d} \Gamma_{h}\left(\mathbf{y}_{h}\right) \mathrm{d} \Gamma_{h}\left(\mathbf{x}_{h}\right) .
\end{aligned}
$$

Before an assembling process, the numerical implementation involves elementary integrals which depend on quantities like $\nabla_{\Gamma_{h}} p_{h \mid \Gamma_{h}}$ locally computed in a basis of each triangle $K[1,2,39,40]$. In the case of some disjoint triangles, the integrals can be computed by a standard quadrature formula. For near interactions, a semi-numerical quadrature formula $[1,8]$ can be used. The convergence rate of the boundary element method is $\mathcal{O}\left(h^{1 / 2}\right)$ since a consistency error [9] involves in the approximation of the exact surface by a polyhedral one. The size of the dense linear system associated to (5) is $(N T+N V)$. The solution to the linear system can be obtained by a Gauss elimination solver or more efficiently by a preconditioned subspace iterative solver accelerated by a "fast multipole method". 


\section{The GENERALIZED IMPEDANCE BOUNDARY-VALUE PROBLEM}

\subsection{The generalized impedance operators}

Let us recall that the transmitted wave can be computed by the Helmholtz integral representation [16]

$$
\forall \mathbf{x} \in \Omega_{1}, u_{1}(\mathbf{x})=\int_{\Gamma}\left(G_{1}(\mathbf{x}, \mathbf{y}) \zeta_{1}(\mathbf{y})-\partial_{\mathbf{n}(\mathbf{y})} G_{1}(\mathbf{x}, \mathbf{y}) p_{1}(\mathbf{y})\right) \mathrm{d} \Gamma(\mathbf{y})
$$

The above relation shows that the interior field can be determined by the interior Cauchy data $\left(\zeta_{1}, p_{1}\right)$. In other words, if the Steklov-Poincaré operator $Y$ is known for the interior problem

$$
\zeta_{1}=Y p_{1}, \quad \text { on } \Gamma
$$

the solution $u_{1}$ can be obtained by a simple calculation of $p_{1}\left(\zeta_{1}\right.$ being computed by Eq. (7)). Unfortunately, $Y$ cannot generally be explicitly computed for a surface. Moreover, even if it would be the case, no numerical gain is made comparatively to the exact integral approach since this operator is a non-local pseudodifferential operator of order one. However, when the interior propagation phenomenon is such that the intensity of the internal field is essentially located near the boundary, the operator $Y$ can be efficiently localized by a high-frequency asymptotic analysis [5]. If $Y_{\ell}$ denotes the differential operator of order $\ell \in \mathbb{N}^{*}$ arising from the localization of $Y$, we can define the approximate densities $\left(\widetilde{\zeta_{1}}, \widetilde{p_{1}}\right)$ linked by the relation: $\widetilde{\zeta_{1}}=Y_{\ell} \widetilde{p_{1}}$, on $\Gamma$. The surface fields $\left(\widetilde{\zeta_{1}}, \widetilde{p_{1}}\right)$ are an approximation of the Cauchy data $\left(\zeta_{1}, p_{1}\right)$. For the sake of conciseness, we do not mention the dependence of $\left(\widetilde{\zeta_{1}}, \widetilde{p_{1}}\right)$ with respect to $\ell$. In electromagnetism [35-37], the operators $Y_{\ell}$ are generally called generalized impedance operators of order $\ell$ (at this step, let us note that these operators are called admittance operators in acoustic scattering; the impedance is defined as the inverse of the admittance; however, we choose here the denomination impedance which is maybe more commonly used in the theory of generalized boundary conditions [36]). They depend on the physical parameters $\alpha, N$ and $k_{1}$ but also on the geometry of the scatterer. In [5], we show that

$$
Y_{\ell}=-\operatorname{div}_{\Gamma}\left(A_{\ell} \nabla_{\Gamma}\right)+\alpha_{\ell}, \quad l=1,2,
$$

where $A_{\ell}$ is a tensor defined on the tangent plane $T_{x}(\Gamma)$ and $\alpha_{\ell}$ a scalar surface field on $\Gamma$. The operator div $\Gamma$ designates the surfacic divergence operator. We can define an approximation $\left(\widetilde{\zeta_{2}}, \widetilde{p_{2}}\right)$ of the exterior Cauchy data $\left(\zeta_{2}, p_{2}\right)$ by using the transmission conditions. The pair $\left(\widetilde{\zeta_{2}}, \widetilde{p_{2}}\right)$ then satisfies the relation

$$
\widetilde{\zeta_{2}}=Y_{\ell} \widetilde{p_{2}}, \text { on } \Gamma \text {. }
$$

Before giving the explicit form of $Y_{\ell}$ for $\ell=1,2$, let us introduce some useful notations linked to the geometry of the interface $\Gamma$. Let $\mathcal{R}$ be the curvature tensor. Its eigenvalues are the principal curvatures $\kappa_{1}$ and $\kappa_{2}$. More precisely, if $R_{1}$ and $R_{2}$ designate the principal curvature radii, we have $\kappa_{j}=1 / R_{j}, j=1,2$. We define $\mathcal{H}=\left(\kappa_{1}+\kappa_{2}\right) / 2$ as the mean curvature and $\mathcal{K}=\kappa_{1} \kappa_{2}$ as the Gauss curvature. Finally, $\Delta_{\Gamma}=\operatorname{div}_{\Gamma} \nabla_{\Gamma} \operatorname{denotes}$ the Laplace-Beltrami operator.

According to [5], the first-order condition corresponds to the choice $A_{1}:=0$ and $\alpha_{1}:=-\alpha\left(i k_{2} N+\mathcal{H}\right)$ and the second-order one to

$$
\left\{\begin{array}{l}
A_{2}:=-\alpha\left(\frac{1}{2 i k_{2} N}\left(\mathbb{I}+\frac{i \mathcal{R}}{k_{2} N}\right)\right) \\
\alpha_{2}:=-\alpha\left(i k_{2} N+\mathcal{H}-\frac{1}{2 i k_{2} N}\left(\mathcal{H}^{2}-\mathcal{K}\right)\left(1+\frac{2 i \mathcal{H}}{k_{2} N}\right)-\frac{1}{4 k_{2}^{2} N^{2}} \Delta_{\Gamma} \mathcal{H}\right),
\end{array}\right.
$$

setting $\mathbb{I}$ as the identity operator of $T_{x}(\Gamma)$. 
Remark 3.1. Higher-order differential operators can be constructed by a recursive process described in [5]. They should lead to more accurate approximations of the Cauchy data. However, they require the use of higherorder boundary element methods which can be difficult to numerically handle and penalize the computational cost. An alternative solution consists in rather using some Padé approximants for computing the symbols of the expansion of the exact impedance operator. This possibility is examined in [3] but still requires further developments.

\subsection{An existence and uniqueness result}

The pair $\left(\widetilde{\zeta_{2}}-\partial_{\mathbf{n}} u_{0 \mid \Gamma}, \widetilde{p_{2}}-u_{0 \mid \Gamma}\right)$ defines a scattered field $w_{2}$ through $(6)$. Therefore, $w_{2}$ satisfies the Helmholtz equation in the exterior domain of propagation $\Omega_{2}$ and the Sommerfeld radiation condition at infinity. The natural question is now the following: if we can define $w_{2 \mid \Gamma}$ and $\partial_{\mathbf{n}} w_{2 \mid \Gamma}$, do we have the two identifications $w_{2 \mid \Gamma}=\widetilde{p_{2}}-u_{0 \mid \Gamma}$ and $\partial_{\mathbf{n}} w_{2 \mid \Gamma}=\widetilde{\zeta_{2}}-\partial_{\mathbf{n}} u_{0 \mid \Gamma}$ ? According to Equation (8), we propose to define $w_{2}$ as the solution to the boundary-value problem

$$
\left\{\begin{array}{l}
\Delta w_{2}+k_{2}^{2} w_{2}=0 \text { in } \Omega_{2}, \\
\left(\partial_{\mathbf{n}}-Y_{\ell}\right) w_{2}=g_{\ell} \text { on } \Gamma, \\
S R C\left(w_{2}\right) .
\end{array}\right.
$$

In formulation (9), the source term $g_{\ell}$ is given by: $g_{\ell}=-\left(\partial_{\mathbf{n}}-Y_{\ell}\right) u_{0}$. Let us prove that the boundary-value problem (9) admits a unique solution under some sufficient conditions, giving hence an answer to the above question under some precised conditions.

In the case of the first-order condition $\left(A_{1}:=0\right)$, the impedance operator is a zeroth-order operator. The boundary condition is a Fourier-Robin condition. The resulting boundary-value problem (9) is classical to solve by considering the space $H_{\text {loc }}^{1}\left(\overline{\Omega_{2}}\right)$. Since the boundary condition is a compact perturbation of the Neumann condition, the Riesz-Fredholm theory applies and the existence of a solution is ensured providing the uniqueness only. The following result holds.

Proposition 3.2. Let $g_{1}$ be given in $H^{-1 / 2}(\Gamma)$. We assume that the mean curvature $\mathcal{H}$ is positive. Then, the exterior boundary-value problem (9) related to the Fourier-Robin impedance boundary condition admits one and only one solution $w_{2}$ in $H_{l o c}^{1}\left(\overline{\Omega_{2}}\right)$ provided

$$
k_{2} \mathcal{H}^{-1} \Im(N)>\left(1+\frac{1}{2 c_{r}^{2} \Im^{2}(N)}\right)^{-1}
$$

Proof. We use a standard argument involving the Rellich lemma [29] to prove the uniqueness. Let $B_{R}$ be a ball centered at the origin with a sufficiently large radius $R$ to enclose $\Omega_{1}$. Setting $\Omega_{R}:=\Omega_{2} \cap B_{R}$, we get

$$
\int_{\Omega_{R}}\left(\Delta w_{2}+k_{2}^{2} w_{2}\right) \overline{w_{2}} \mathrm{~d} \Omega_{R}=0
$$

By using the usual Green formula, the above variational equation modifies to

$$
\int_{\Omega_{R}}\left(k_{2}^{2}\left|w_{2}\right|^{2}-\left|\nabla w_{2}\right|^{2}\right) \mathrm{d} \Omega_{R}=\int_{\Gamma} \partial_{\mathbf{n}} w_{2} \overline{w_{2}} \mathrm{~d} \Gamma-\int_{S_{R}} \partial_{r} w_{2} \overline{w_{2}} \mathrm{~d} S_{R}
$$

where $S_{R}:=\partial B_{R}$ denotes the boundary of $B_{R}$. Since the integral on $\Omega_{R}$ is real, we get

$$
\Im \int_{\Gamma} \partial_{\mathbf{n}} w_{2} \overline{w_{2}} \mathrm{~d} \Gamma=\Im \int_{\Gamma} \alpha_{1}\left|w_{2}\right|^{2} \mathrm{~d} \Gamma=\Im \int_{S_{R}} \partial_{r} w_{2} \overline{w_{2}} \mathrm{~d} S_{R} .
$$


Let us remark that $\Im\left(-\alpha_{1}\right)=k_{2} \Re(\alpha N)+\mathcal{H} \Im(\alpha), \Re(\alpha N)>0$ and $\Im(\alpha)<0$. It results that the imaginary part of $\alpha_{1}$ is negative if

$$
k_{2} \Re(\alpha N)+\mathcal{H} \Im(\alpha)>0 \Leftrightarrow k_{2}>-\mathcal{H} \frac{\Im(\alpha)}{\Re(\alpha N)} .
$$

Noticing that $\Re(\alpha)=-\gamma_{1}^{-1} \Im(\alpha)$, with $\gamma_{1}=\delta / \omega$, we conclude that $\Re(\alpha N)=-\Im(\alpha)\left(\Im(N)+\gamma_{1}^{-1} \Re(N)\right)$. As a consequence, inequality (11) reads

$$
k_{2}>\frac{\mathcal{H}}{\Im(N)+\gamma_{1}^{-1} \Re(N)} .
$$

Moreover, using the definition of the refractive index $N$, a simple calculation gives: $\Re(N) \Im(N)=\gamma_{1} /\left(2 c_{r}^{2}\right)$. Therefore, relation (11) can be simplified as

$$
k_{2} \mathcal{H}^{-1} \Im(N)>\left(1+\frac{1}{2 c_{r}^{2} \Im^{2}(N)}\right)^{-1} .
$$

Consequently, since we get

$$
\Im\left(\int_{S_{R}} \partial_{r} w_{2} \overline{w_{2}} \mathrm{~d} S_{R}\right) \leq 0,
$$

the uniqueness is then classically obtained by using the Sommerfeld condition and the Rellich lemma.

In [5], we describe how to approximate the Steklov-Poincaré operator in a consistent way for the interior problem by an asymptotic analysis with respect to the high-frequency parameter $\left|k_{1}\right|=k_{2}|N| \gg 1$. In this approach, another possibility is to consider a high refraction index $N$, i.e. $|N| \gg 1$, to get the same generalized impedance boundary conditions. As a consequence, condition $|N| \gg 1$ is a first condition to impose to ensure the validity of the approximation of the transmission acoustic scattering problem using an impedance boundary condition. Moreover, as proposed in Proposition 3.2, a sufficient condition to have a well-posed problem is

$$
k_{2} \mathcal{H}^{-1} \Im(N)>\left(1+\frac{1}{2 c_{r}^{2} \Im^{2}(N)}\right)^{-1} .
$$

Hence, the existence and uniqueness to the approximate problem is proved if

$$
k_{2} \Im(N) \mathcal{H}^{-1}>\left(1+\frac{1}{2 c_{r}^{2} \Im^{2}(N)}\right)^{-1} \text { and }|N| \gg 1 .
$$

In the numerical study of the validity of the approximate model using a Fourier-Robin (order 1/2) boundary condition

$$
\partial_{\mathbf{n}} w_{2}+i \alpha k_{2} N w_{2}=g_{1 / 2}, \quad \text { on } \Gamma,
$$

in the context of two-dimensional electromagnetism, Wang [36,41] has given two quantitative validity conditions of the model

$$
k_{2} R \Im(N)>C_{1} \text { and }|N|>C_{2},
$$

for a circular cylinder $\Omega_{1}$ of radius $R$. The real positive constants $C_{1}$ and $C_{2}$ are obtained by fixing a tolerance on the computation of the far-field. These relations have next been extended to a second-order generalized impedance boundary condition by Senior and Volakis [36]. To the best of the authors' knowledge, no theoretical justification on the origin of conditions (16) has been given. Moreover, these conditions have been extrapolated in the framework of the three-dimensional acoustics. A possible explanation is that only the Fourier-Robin boundary condition (15) is usually used in acoustics. However, this condition does not take the geometry of the scatterer into account. When the well-posedness of the boundary-value problem is examined, then only conditions involving the physical parameters of the problem are needed during the proof of the existence and uniqueness result [35]. Hence, conditions (14) can be seen as a qualitative justification of Wang's conditions (16) 
extended to the case of the acoustic scattering by an arbitrarily shaped convex body. In the situation where the hypothesis $\mathcal{H}>0$ is not satisfied everywhere, the global property (13) may still be fulfilled but a sufficient condition cannot be so explicitly stated. This remains an interesting issue to investigate in details.

The second-order impedance boundary condition is defined by the operator $Y_{2}$. The boundary-value problem is a non-standard scattering problem (sometimes also called a Ventcel problem). A well-adapted functional framework is given by the Fréchet space $H_{\text {loc }}^{1,1}\left(\overline{\Omega_{2}}, \Gamma\right)=\left\{u \in H_{\text {loc }}^{1}\left(\overline{\Omega_{2}}\right),\left.\quad u\right|_{\Gamma} \in H^{1}(\Gamma)\right\}$. According to [40], one gets the following theorem.

Theorem 3.3. Let us assume that $g_{2} \in H^{-1}(\Gamma)$ and that

$$
\Im\left(A_{2}(x) \boldsymbol{\xi} \cdot \overline{\boldsymbol{\xi}}\right) \leq 0, \forall x \in \Gamma, \forall \boldsymbol{\xi} \in T_{x}(\Gamma), \quad \text { and } \Im\left(\alpha_{2}(x)\right) \leq 0, \forall x \in \Gamma
$$

Then, the boundary-value problem (9) admits a unique solution $w_{2}$ in $H_{l o c}^{1,1}\left(\overline{\Omega_{2}}, \Gamma\right)$. Moreover, if $g_{2} \in H^{s}(\Gamma)$, $s \geq-1$, then we have: $w_{2} \in H_{\text {loc }}^{s+5 / 2}\left(\overline{\Omega_{2}}\right)$.

Thus, the well-posedness of problem (9) is ensured provided $A_{2}$ and $\alpha_{2}$ satisfy conditions (17). As far as the numerical solution of (9) is concerned, we intend to consider the conditions involving (1)-(2). The following proposition holds.

Proposition 3.4. Let us assume that $\Omega_{1}$ is a convex domain and that we have the inequality

$$
\frac{\Delta_{\Gamma} \mathcal{H}}{4}+\mathcal{H}\left(\mathcal{H}^{2}-\mathcal{K}\right) \geq 0
$$

Moreover, we suppose that $\left.\gamma_{1} \in\right] \sqrt{3},+\infty[$. Then, conditions (17) are satisfied provided

$$
k_{2} \mathcal{H}^{-1} \Im(N)>\frac{1}{2}\left(1+\frac{1}{2 c_{r}^{2} \Im^{2}(N)}\right)^{-1}\left(1+\sqrt{\left(1+\frac{2\left(\mathcal{H}^{2}-\mathcal{K}\right)}{\mathcal{H}^{2}} \frac{\Im(N)^{2}}{|N|^{2}}\left(1-\frac{1}{4 c_{r}^{4} \Im^{4}(N)}\right)\right)}\right)
$$

and

$$
k_{2} \Im(N) \min _{x \in \Gamma} R_{j}(x)>\frac{2}{c_{r}^{2}|N|^{2}}\left(1-\frac{1}{2 c_{r}^{2} \Im^{2}(N)}\right)^{-1}
$$

Proof. We have already seen that it is sufficient to fulfill that $\Im\left(A_{2} \boldsymbol{\xi} \cdot \overline{\boldsymbol{\xi}}\right) \leq 0$. If $\Omega_{1}$ is convex, the eigenvalues of the curvature operator $\mathcal{R}$ are positive and we have $\mathcal{R} \boldsymbol{\xi} \cdot \overline{\boldsymbol{\xi}} \geq 0$. Moreover, a straightforward calculation yields

$$
\Im\left(A_{2}(x) \boldsymbol{\xi} \cdot \overline{\boldsymbol{\xi}}\right)=\frac{1}{2 k_{2}} \Re\left(\frac{\alpha}{N}\right)|\boldsymbol{\xi}|^{2}-\frac{1}{2 k_{2}^{2}} \Im\left(\frac{\alpha}{N^{2}}\right) \mathcal{R} \boldsymbol{\xi} \cdot \overline{\boldsymbol{\xi}} .
$$

Without any specific hypothesis, $\Im\left(\alpha / N^{2}\right)$ is negative and we get $\left.\Re\left(\frac{\alpha}{N}\right)>0 \Longleftrightarrow \gamma_{1} \in\right] 0, \sqrt{3}\left[, \Re\left(\frac{\alpha}{N}\right)<0 \Longleftrightarrow\right.$ $\left.\gamma_{1} \in\right] \sqrt{3},+\infty\left[\right.$. Hence, we deduce that if $\left.\gamma_{1} \in\right] 0, \sqrt{3}\left[\right.$, then we have: $\Im\left(A_{2}(x) \boldsymbol{\xi} \cdot \overline{\boldsymbol{\xi}}\right)>0$. This implies that we cannot ensure the uniqueness of the solution if $\left.\gamma_{1} \in\right] 0, \sqrt{3}$. It does not mean that the solution is not unique since the inequality $\Im\left(A_{2}(x) \boldsymbol{\xi} \cdot \overline{\boldsymbol{\xi}}\right) \leq 0$ is only a sufficient condition. However, we will later see that the case $\left.\gamma_{1} \in\right] 0, \sqrt{3}[$ (corresponding to a weak absorbing medium) yields an inaccurate approximate model. Therefore, we restrict our attention to the case $\left.\gamma_{1} \in\right] \sqrt{3},+\infty\left[\right.$. Then, $\Im\left(A_{2}(x) \boldsymbol{\xi} \cdot \overline{\boldsymbol{\xi}}\right) \leq 0$ if and only if we have

$$
k_{2} R_{j}(x)>\frac{\Im\left(\alpha / N^{2}\right)}{\Re(\alpha / N)}, \quad j=1,2 .
$$


A direct calculation gives $\Im\left(\alpha N^{-2}\right)=\Re(\alpha) \Im\left(N^{-2}\right)+\Im(\alpha) \Re\left(N^{-2}\right)$. Since $\Re(\alpha)=-\gamma_{1}^{-1} \Im(\alpha)$, we get $\Im\left(\frac{\alpha}{N^{2}}\right)=\frac{\Im(\alpha)}{|N|^{4}}\left(\Re\left(N^{2}\right)+\gamma_{1}^{-1} \Im\left(N^{2}\right)\right)$, which can be further reduced to

$$
\Im\left(\frac{\alpha}{N^{2}}\right)=\frac{2 \Im(\alpha)}{c_{r}^{2}|N|^{4}}
$$

using $\Re\left(N^{2}\right)=\gamma_{1}^{-1} \Im\left(N^{2}\right)$ and $\Im\left(N^{2}\right)=\gamma_{1} / c_{r}^{2}$.

Let us consider now the term $\Re(\alpha / N)$. Using $\Re\left(\frac{\alpha}{N}\right)=\frac{1}{|N|^{2}}(\Re(\alpha) \Re(N)+\Im(\alpha) \Im(N))$, $\Re(\alpha)=-\gamma_{1}^{-1} \Im(\alpha)$ and $2 c_{r}^{2} \Im(N)=\gamma_{1}(\Re(N))^{-1}$, we obtain

$$
\Re\left(\frac{\alpha}{N}\right)=\frac{\Im(\alpha) \Im(N)}{|N|^{2}}\left(1-\frac{1}{2 c_{r}^{2} \Im^{2}(N)}\right) .
$$

Then, by plugging (22) and (23) into $(21)$ when $\left.\gamma_{1} \in\right] \sqrt{3},+\infty\left[\right.$, we see that $\Im\left(A_{2}(x) \boldsymbol{\xi} \cdot \overline{\boldsymbol{\xi}}\right) \leq 0$ if and only if the following inequality holds

$$
k_{2} \Im(N) \min _{x \in \Gamma} R_{j}(x)>\frac{2}{c_{r}^{2}|N|^{2}}\left(1-\frac{1}{2 c_{r}^{2} \Im^{2}(N)}\right)^{-1} .
$$

Let us study the sign of $\alpha_{2}$. Since $\Im\left(\alpha N^{2}\right)$ and $\Im\left(\alpha / N^{2}\right)$ have the same signs, the following inequality holds:

$$
\frac{\Im\left(\frac{\alpha}{N^{2}}\right)}{k_{2}^{2}}\left(\frac{\Delta_{\Gamma} \mathcal{H}}{4}+\mathcal{H}\left(\mathcal{H}^{2}-\mathcal{K}\right)\right) \leq 0,
$$

under the assumption $\Delta_{\Gamma} \mathcal{H} / 4+\mathcal{H}\left(\mathcal{H}^{2}-\mathcal{K}\right) \geq 0$. Hence, the inequality $\Im\left(\alpha_{2}\right) \leq 0$ is satisfied if we have

$$
\Re(\alpha N) k_{2}^{2}+\mathcal{H} \Im(\alpha) k_{2}+\frac{\left(\mathcal{H}^{2}-\mathcal{K}\right)}{2} \Re\left(\frac{\alpha}{N}\right) \geq 0 .
$$

Now, let us observe that $\Re(\alpha N)$ and $\Re(\alpha / N)$ have the same signs. Hence, if $\left.\gamma_{1} \in\right] \sqrt{3},+\infty[$, Equation (24) is equivalent to

$$
P\left(k_{2}\right)=k_{2}^{2}+\frac{\mathcal{H} \operatorname{Im}(\alpha)}{\Re(\alpha N)} k_{2}+\frac{\left(\mathcal{H}^{2}-\mathcal{K}\right)}{2 \Re(\alpha N)} \Re\left(\frac{\alpha}{N}\right) \geq 0 .
$$

A computation of the discriminant $D$ associated to the above polynomial $P$ shows that $D>0$ if $\left.\gamma_{1} \in\right] \sqrt{3},+\infty[$. Hence, $P$ admits two distinct real roots $k_{2}^{ \pm}$of opposite sign. The positive root $k_{2}^{+}$is given by

$$
k_{2}^{+}=-\frac{\mathcal{H} \Im(\alpha)}{2 \Re(\alpha N)}\left(1+\left(1-\frac{2\left(\mathcal{H}^{2}-\mathcal{K}\right) \Re\left(\alpha N^{-1}\right) \Re(\alpha N)}{\mathcal{H}^{2} \Im(\alpha)^{2}}\right)^{1 / 2}\right) .
$$

Some simple calculations allow to show that

$$
\Re(\alpha N) \Re\left(\frac{\alpha}{N}\right)=\Re(\alpha)^{2} \Re(N) \Re\left(\frac{1}{N}\right)+\Im(\alpha)^{2} \Im(N) \Im\left(\frac{1}{N}\right)-\Im(\alpha) \Re(\alpha)\left[\Im(N) \Re\left(\frac{1}{N}\right)+\Im\left(\frac{1}{N}\right) \Re(N)\right] .
$$

Moreover, since $-\gamma_{1} \Re(\alpha)=\Im(\alpha)$, we also have $\frac{\Re(\alpha N) \Re\left(\alpha N^{-1}\right)}{\Im(\alpha)^{2}}=\left[\frac{1}{\gamma_{1}^{2}} \Re(N) \Re\left(\frac{1}{N}\right)+\Im(N) \Im\left(\frac{1}{N}\right)\right]$. Finally, since $\Re(1 / N)=\Re(N) /|N|^{2}$ and $\Im(1 / N)=-\Im(N) /|N|^{2}$, we get $\frac{\Re(\alpha N) \Re\left(\alpha N^{-1}\right)}{\Im(\alpha)^{2}}=-\frac{\Im(N)^{2}}{|N|^{2}}\left[1-\frac{1}{4 c_{r}^{4} \Im^{4}(N)}\right]$. Therefore, (25) is satisfied if $k_{2} \geq k_{2}^{+}$or in other words if

$$
k_{2} \mathcal{H}^{-1} \Im(N)>\frac{1}{2}\left(1+\frac{1}{2 c_{r}^{2} \Im^{2}(N)}\right)^{-1}\left(1+\sqrt{\left(1+\frac{2\left(\mathcal{H}^{2}-\mathcal{K}\right)}{\mathcal{H}^{2}} \frac{\Im(N)^{2}}{|N|^{2}}\left(1-\frac{1}{4 c_{r}^{4} \Im^{4}(N)}\right)\right)}\right) .
$$


In the case of a sphere, the condition (19) associated to the second-order condition is identical to the one obtained for the first-order condition and condition (18) is fulfilled. For a regular surface, quantity $\left(\mathcal{H}^{2}-\mathcal{K}\right) / \mathcal{H}^{2}=\left(\kappa_{1}-\kappa_{2}\right)^{2} /\left(\kappa_{1}+\kappa_{2}\right)^{2}$ is generally quite small and always lower than 1 . Similarly, expression $\Im(N)^{2} /|N|^{2}$ is inferior to 1 . Hence, condition (19) is not much more restrictive than (10). Condition (20) is not so restrictive as it looks like. Indeed, the approximate model is associated to some physical parameters such that $\Im(N) \gg 1$ and $|N| \gg 1$. This implies that in (20), the right hand-side tends to be small while the left hand-side is relatively large. If we compare the sufficient conditions in Propositions 3.2 and 3.4, it seems at first sight that the second-order condition is more restrictive than the first-order one. However, these conditions must be coupled with the asymptotic one: $|N| \gg 1$. Hence, the conditions of Proposition 3.4 are satisfied for a larger range of parameters $\left(k_{2}, N, \alpha\right)$ than in Proposition 3.2. We will see in the following section that the same conclusion holds when considering the validity domain of each impedance boundary condition. Finally, condition $\gamma_{1} \geq \sqrt{3}$ must be satisfied when applying the second-order condition to obtain a sufficiently small error. From a practical point of view, it is not a real restriction as seen in the following section.

\subsection{Numerical validity domain}

It is not surprising that the impedance boundary-value problem approximates the exact problem with a good accuracy for a suitable set of parameters. Indeed, the impedance boundary condition arises from an approximation of an exact condition. This approximation is justified for a sufficiently absorbing object [5]. In this situation, the main part of the energy of the interior field is localized near the interface. Therefore, the localization of the exact boundary operator is valid and leads to an accurate approximate operator.

To precise the validity domain of the method, we consider the scattering problem of a plane wave $u_{0}$ which strikes the unitary ball centered at the origin. More precisely, we take $u_{0}=\exp \left(-i k_{2} x_{1}\right)$. We define the far-field pattern given by the Sonar cross section (SCS)

$$
\operatorname{SCS}(\theta)=10 \log _{10}\left(4 \pi|a(\theta)|^{2}\right) \quad(\mathrm{dB}),
$$

where $a(\theta)$ denotes the diffusion amplitude of the scattered field in the direction $\theta=(\cos \theta \cos \varphi, \sin \theta \cos \varphi, \sin \varphi)$, setting $(\theta, \varphi)$ as the spherical angular coordinates on the unit sphere. We represent the relative error on the SCS for the $L^{2}\left(S_{R}\right)$-norm, where $S_{R}$ designates the sphere centered at the origin and with a radius $R$ large enough to have a suitable representation of the far-field.

Let us choose $c_{r}=0.75, \rho_{r}=1.2$ and $\gamma_{1}=2$. The wavenumber $k_{2}$ is equal to 15 . In Figure 1 , we can observe that the bistatic SCS is more accurate for the second-order condition (the relative error is $0.5 \%$ ) than for the first-order one (a relative error of $11.6 \%$ ). Similar results have been reported in [5].

Now, we want to compute an approximate SCS with an error lower than $2 \%$. In Figure 2, we represent the evolution of the relative error according to $k_{2}$ (for $c_{r}=0.75, \rho_{r}=1.2$ and $\gamma_{1}=2$ ). We see that the approach is a high-frequency method: the error decreases as the wavenumber increases. Moreover, we can observe that the first-order impedance boundary condition leads to a relative error on the SCS which stabilizes around $10 \%$. In the case of the second-order condition, the relative error is lower than $2 \%$ as soon as $k_{2} \geq 6.6$.

We consider in Figure 3 the variations of the error with respect to the relative velocity $c_{r}$. To this end, we take $k_{2}=6.6, \rho_{r}=1.2, \gamma_{1}=2$. The quality of the approximate solution is better as $c_{r}$ decreases. To get an error lower than $2 \%$, we see that $c_{r}$ must be lower than 0.4 for the first-order condition and than 0.75 for the second-order condition. In fact, the relative velocity $c_{r}$ is equal to $c_{1} / c_{2}$, where $c_{1}$ and $c_{2}$ are respectively the velocity in the scatterer and in the external medium. In both cases, the accuracy of the method is guaranteed as long as $c_{1}$ is strictly lower than $c_{2}$. We have already mentioned the fact that the proposed approach is valid in the case where the interior propagation is mainly localized near the interface $\Gamma$. This is effectively the case when $c_{1} / c_{2} \ll 1: c_{r}$ must be small enough to ensure a good accuracy. This last point was already emphasized in Proposition 3.4 fixing any of the parameters and varying $c_{r}$. This latter parameter must be small enough to get the uniqueness of the approximate solution. A wider applicability of the second-order condition compared to the first-order condition is observed. 


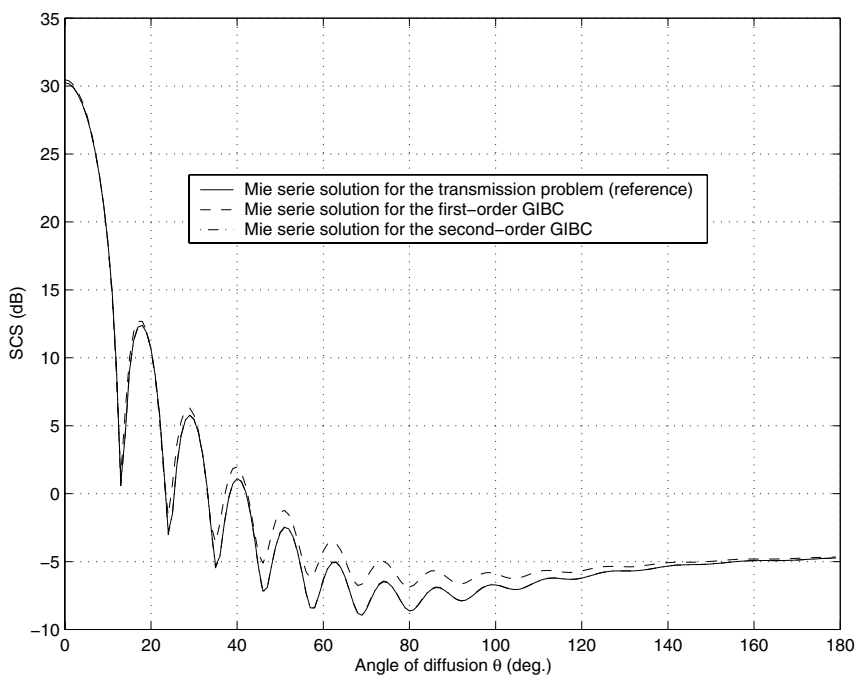

FIGURE 1. Far field patterns using a first and a second-order generalized impedance boundary condition.

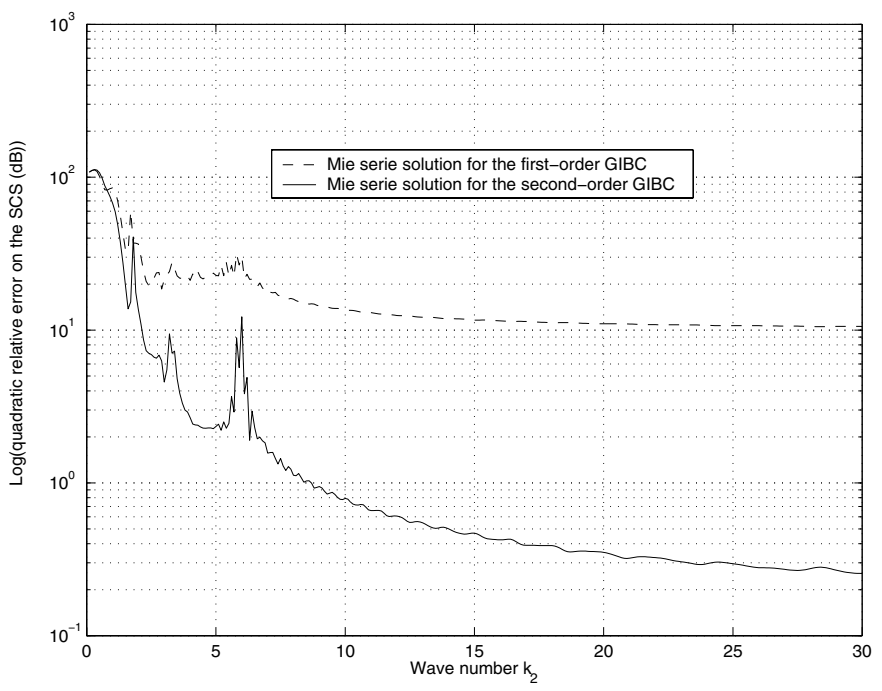

Figure 2. Computation of the relative error on the SCS according to $k_{2}$.

We consider now the relative error as a function of $\rho_{r}$. Let us fix $k_{2}=6.6, c_{r}=0.75, \gamma_{1}=2$. We observe in Figure 4 that the relative error is maximal (around $2 \%$ ) for $\rho_{r}=1.3$ for the second-order condition. In the case of the first-order condition, the error is relatively important for any value of $\rho_{r}$. We observe that the influence of the relative density on the error is not really significant for the second-order condition.

Finally, we consider that $\gamma_{1}=\delta / \omega$ varies and we choose $k_{2}=6.6, c_{r}=0.75$ and $\rho_{r}=1.2$. Figure 5 shows that the relative error decreases as $\gamma_{1}$ increases. Moreover, the error is lower than $2 \%$ for the second-order condition when $\gamma_{1} \geq 2.5$. This is a very interesting property since we have proved that the problem is well-posed when $\gamma_{1}>\sqrt{3}(\approx 1.73)$ 


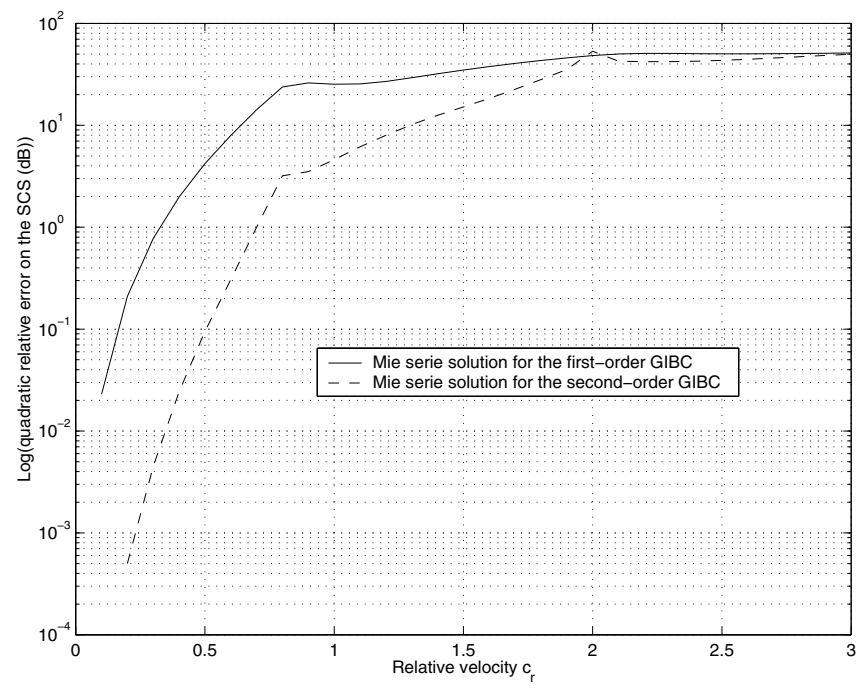

FiguRE 3. Computation of the relative error on the SCS according to $c_{r}$.

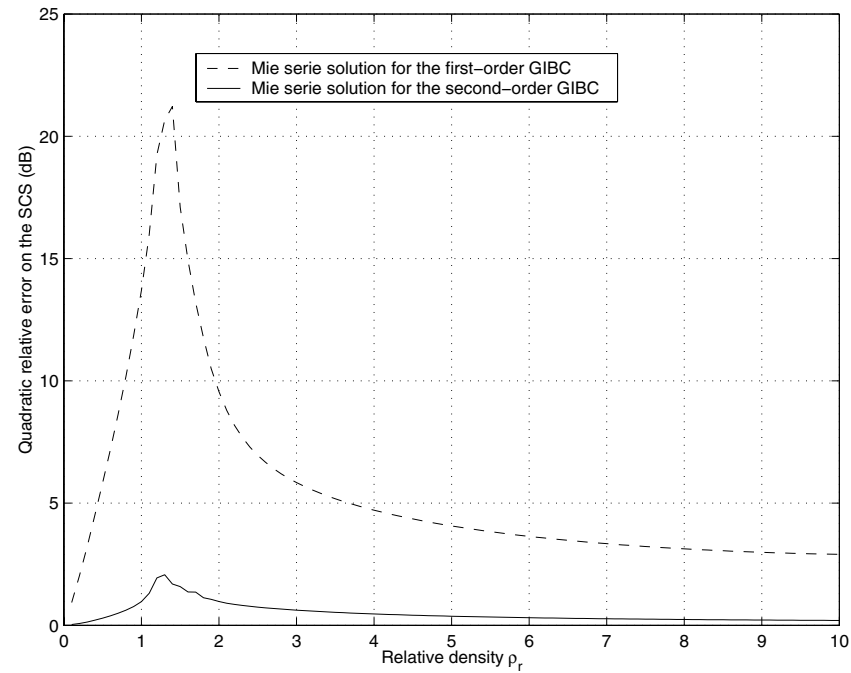

Figure 4. Computation of the relative error on the SCS according to $\rho_{r}$.

The validity analysis makes our previous theoretical study consistent. We obtain a condition linking $k_{2}, N$ and the dimensions of the scatterer to get an error lower than $2 \%$ for a given impedance condition. For the first-order condition, we have $k_{2} R \Im(N) \geq 13.9$ and $|N| \geq 3.8$, while for the second-order condition we show that $k_{2} R \Im(N) \geq 7$ and $|N| \geq 2$. Such conditions are similar to those involved for the well-posedness of the approximate boundary-value problem. Moreover, they are close to the conditions originally derived for the two-dimensional problems [36].

Two significant aspects result from this analysis. The first interesting point is that one can construct an integral equation involving only one unknown surface field, the second one being determined by the impedance condition. Secondly, we see that the use of the microlocal analysis is efficient if $\left|k_{1}\right|$ is large enough. In this 


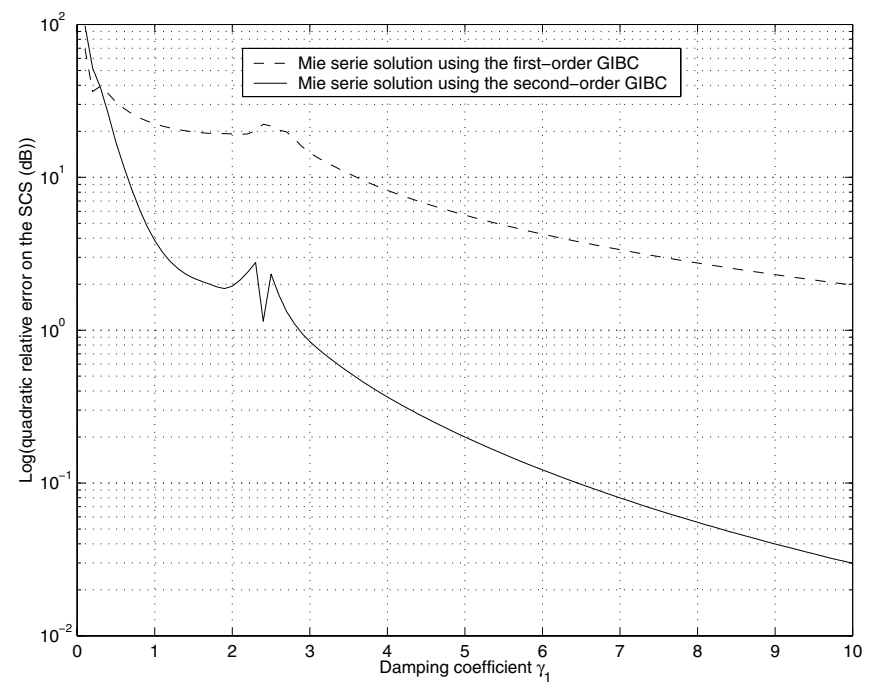

Figure 5. Computation of the relative error on the SCS according to $\gamma_{1}$.

situation, a method which would only require the solution to the exterior boundary-value problem would be interesting for high interior wavenumber. Indeed, in this case, one would have in almost situations a larger wavelength $\lambda_{2}$ in the exterior domain. Since the size of the mesh involved during the approximation of an integral equation is a fraction of the smallest wavelength, one can expect to have a better convergence of a numerical method for solving the approximate model rather than for the exact problem at a given resolution. Since the second-order condition is more efficient, we focus our study on this condition in the above section.

\section{INTEGRAL EQUATION FORMULATION AND NUMERICAL EXPERIMENTS}

This section is devoted to the study of an integral formulation which makes use of the local generalized impedance boundary operator. In a first part, we present the formulation that we designate by combined integral equation (CIE) for the sake of conciseness. In a second part, its numerical approximation is described precising its numerical advantages as compared to the standard integral equation (IE) method. We finally report some numerical tests. As in the case of a spherical scatterer, the CIE method gives accurate results for an ellipsoidal scatterer and provides an interesting alternative to the fully coupled IE formulation.

\subsection{The integral equation formulation (CIE)}

The selected strategy is based on the integral formulation proposed by Vernhet [39,40]. We do not develop all the details of the procedure and refer to $[39,40]$ for completeness. Let us introduce $w_{1}$ as the solution to the non-standard interior boundary-value problem associated to (9) which reads

$$
\left\{\begin{array}{l}
\Delta w_{1}+k_{2}^{2} w_{1}=0 \text { in } \Omega_{1} \\
\left(\partial_{\mathbf{n}}-Y_{\ell}\right) w_{1}=g_{\ell} \text { on } \Gamma .
\end{array}\right.
$$

This problem is purely artificial and $w_{1}$ does not represent an acoustic field which actually exists. Nevertheless by applying the results of [40], we can prove that it is well-posed. To introduce $w_{1}$ allows to use the potential theory [28] to derive the CIE. Let us define $w$ by: $w_{\mid \Omega_{j}}:=w_{j}$, for $j=1,2$. We also consider the densities $\widetilde{m} \in H^{-1 / 2}(\Gamma)$ and $\widetilde{j} \in H^{1}(\Gamma)$ and the Lagrange multiplier $\widetilde{l} \in H^{1}(\Gamma) \widetilde{m}:=\left[\partial_{\mathbf{n}} w\right], \quad \widetilde{j}:=[w]$ and $\tilde{l}:=\frac{w_{1}+w_{2}}{2}$. 
Following [39,40], a suitable Fredholm integral formulation of the first kind adapted to problem (9) (and called here combined integral equation (CIE)) consists in finding $(\widetilde{m}, \widetilde{j}, \widetilde{l}) \in \Theta$ solution to the saddle point problem

$$
\begin{aligned}
& \widetilde{a}((\widetilde{m}, \widetilde{j}),(m, j))+\int_{\Gamma} \widetilde{l}\left(m-Y_{2}^{T} j\right) \mathrm{d} \Gamma=\int_{\Gamma} g_{2} j \mathrm{~d} \Gamma, \\
& \int_{\Gamma} l\left(\widetilde{m}-Y_{2} \widetilde{j}\right) \mathrm{d} \Gamma=0, \forall(m, j, l) \in \Theta,
\end{aligned}
$$

where $\Theta$ is the product space defined by $\Theta:=H^{-1 / 2}(\Gamma) \times H^{1}(\Gamma) \times H^{1}(\Gamma)$. The bilinear form $\widetilde{a}(.,$.$) is such that$

$$
\widetilde{a}((\widetilde{m}, \widetilde{j}),(m, j)):=-\left\langle V_{2} \widetilde{m}, m\right\rangle+\left\langle N_{2} \widetilde{j}, m\right\rangle+\left\langle N_{2} j, \widetilde{m}\right\rangle+\left\langle D_{2} \widetilde{j}, j\right\rangle
$$

with $\left\langle D_{2} \widetilde{j}, j\right\rangle:=c_{2}(\widetilde{j}, j)-d_{2}(\widetilde{j}, j)$ and

$$
\int_{\Gamma} l Y_{2} \widetilde{j} \mathrm{~d} \Gamma=\mathcal{A}_{2}(\widetilde{j}, l):=\int_{\Gamma}\left(A_{2} \nabla_{\Gamma} l \cdot \nabla_{\Gamma} \widetilde{j}+\alpha_{2} \widetilde{l j}\right) \mathrm{d} \Gamma .
$$

The existence and uniqueness of the solution to system (27) is based on the statement of a Babuška inf-sup condition.

\subsection{Boundary element approximation and numerical experiments}

The boundary element approximation of the unknown fields is given by $\left(\widetilde{m_{h}}, \tilde{j_{h}}, \widetilde{l_{h}}\right)$ in $\left(V_{h}\right)^{3}$. At first sight, the dimension of the approximation space is triple compared to the one required for a Neumann problem. Fortunately, a mass lumping process [39,40] allows to avoid this problem by keeping $\widetilde{j_{h}}$ as the only unknown. Hence, the computational burden is similar to the one arising from the solution of a Neumann problem and the approach leads to the solution of a linear system of size $N V_{\lambda_{2}}^{\mathrm{CIE}}$. The memory storage of the CIE method seems a priori three times smaller than for the IE formulation of Section 2 (if we consider that $N T^{\mathrm{IE}} \approx 2 N V^{\mathrm{IE}}$ ). However, one must keep in mind that the discretization of the IE formulation is made according to the smallest wavelength $\min \left(\lambda_{1}, \lambda_{2}\right)$. This implies that if $\lambda_{1} \ll \lambda_{2}$, we have $N V_{\lambda_{2}}^{\mathrm{CIE}} \ll N V_{\lambda_{1}}^{\mathrm{IE}}$ and we should obtain a faster convergence of the boundary element method for solving the CIE formulation. A difficulty concerns the construction of a suitable $\mathcal{P}_{1}$-approximation of the curvature tensor $\mathcal{R}$ in a basis of the tangent plane at a node of the mesh. To this end, we adapt the approach established in [2]. Some numerical experiments (not reported here) using formulation (27) show that the theoretical rate of convergence $[39,40] \mathcal{O}\left(h^{1 / 2}\right)$ of the CIE is not affected by the approximation of $\mathcal{R}$.

The benchmark computations are given by the exact solution computed by its Mie series for the unit sphere or by the IE (with a sufficiently refined mesh) otherwise. The first test case consists in illuminating the unit sphere by an incident plane wave of direction $(-1,0,0)$ for a wavenumber $k_{2}=15$. The physical parameters are $c_{r}=\rho_{r}=1.5$ and $\gamma_{1}=10$. The SCS computations are depicted in Figure 6 . The relative quadratic error on the SCS between the exact analytic Mie solutions for the transmission problem and the generalized impedance problems is $0.1 \%$. This ensures the validity of the approximate model: the generalized impedance boundary value well modelled the transmission problem. For a given resolution, the size $N V_{\lambda_{2}}^{\mathrm{CIE}}$ of the linear system associated to the CIE is three times smaller than the size $N V_{\min \left(\lambda_{1}, \lambda_{2}\right)}^{\mathrm{IE}}$ of the system issued from the discretization of the IE formulation. This reduction of the computational cost is due to the mass lumping process arising from the boundary element method. Now, let us analyze the influence of the mesh resolution on the convergence of the two integral methods (IE and CIE). We present in Table 1 the quadratic relative error on the SCS for several meshes. It appears that the CIE formulation has a better convergence rate than the IE formulation. Moreover, the IE approach requires a refined mesh for a small mesh step to get a satisfactory accuracy. These observations are completely in accordance with the above remarks concerning the wavelength dependence of the sizes $N V_{\lambda_{2}}^{\mathrm{CIE}}$ and $N V_{\min \left(\lambda_{1}, \lambda_{2}\right)}^{\mathrm{IE}}$ of the matrices. Indeed, we have considered a smaller wavelength in the interior domain $\left(\left|k_{1}\right|=30\right)$ than in the exterior domain $\left(k_{2}=15\right)$. Hence, the CIE method seems very attractive in the case of a high refraction coefficient. 

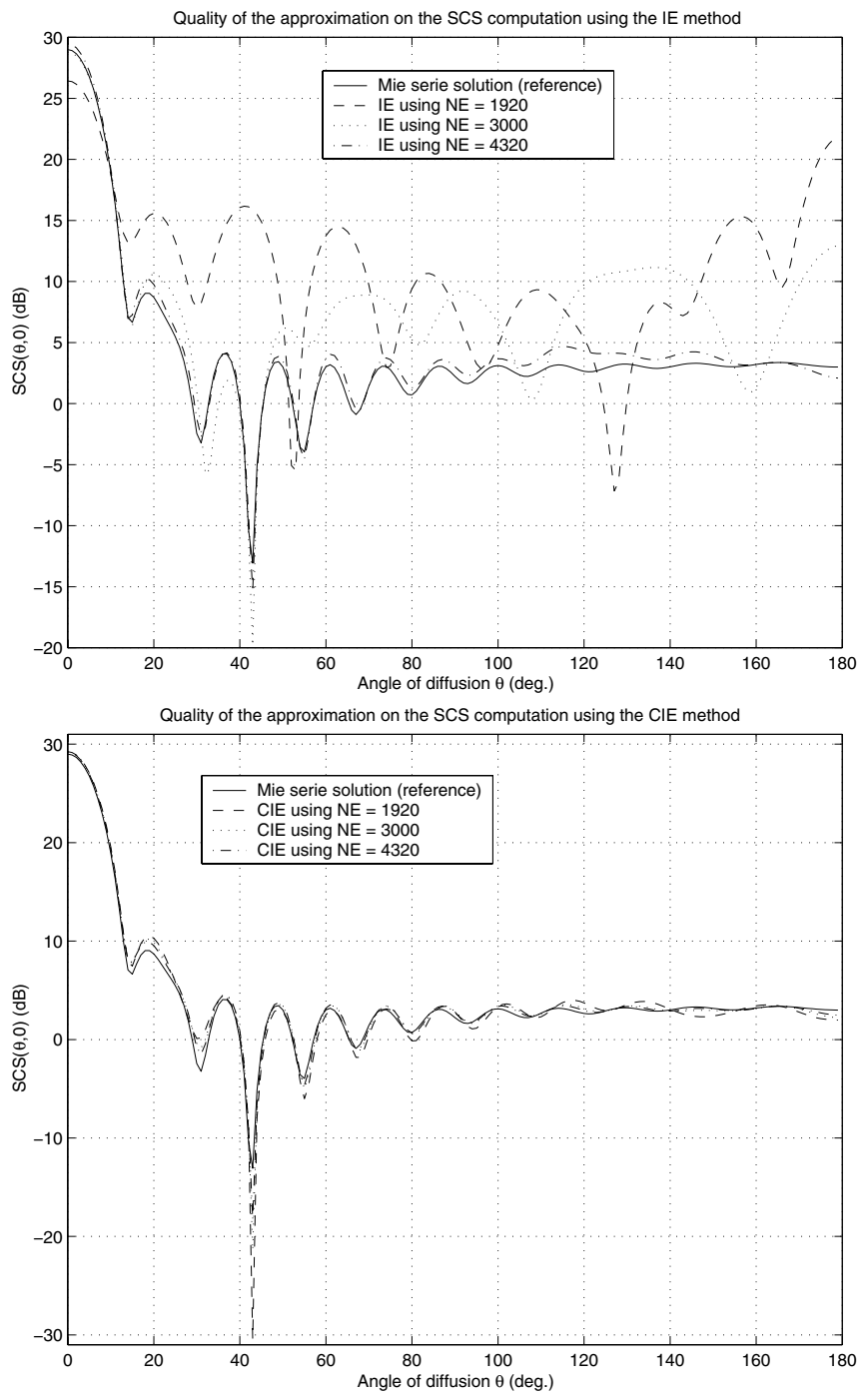

Figure 6. Computation of the SCS (dB) by the IE and CIE methods for the unit sphere.

TABLE 1. Quadratic relative error on the SCS between the exact Mie serie solution and the IE or CIE solution vs. the number $N E$ of edges of the finite element mesh.

\begin{tabular}{|l|l|l|l|l|l|}
\hline NE & 750 & 1080 & 1920 & 3000 & 4320 \\
\hline Quadratic relative error (IE) (\%) & 429 & 259 & 117 & 71 & 28 \\
\hline Quadratic relative error (CIE) (\%) & 76 & 60 & 17 & 13 & 9 \\
\hline
\end{tabular}

TABLE 2. Quadratic relative error on the SCS vs. the number $N E$ of edges.

\begin{tabular}{|l|l|l|l|l|}
\hline NE & 750 & 1080 & 1920 & 3000 \\
\hline Quadratic relative error (IE) (\%) & 66 & 10 & 2 & 0 \\
\hline Quadratic relative error (CIE) (\%) & 18 & 13 & 8 & 6 \\
\hline
\end{tabular}



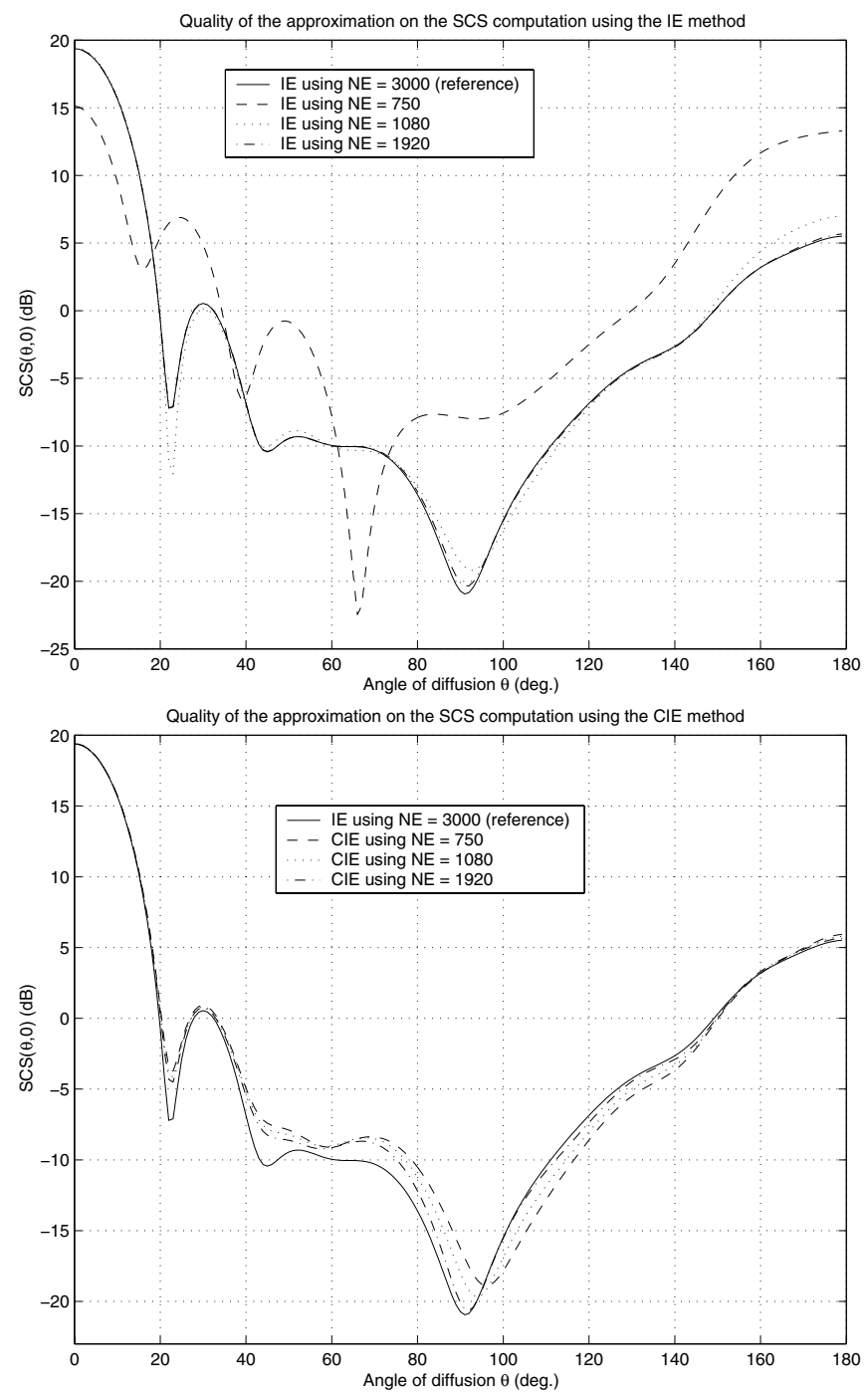

Figure 7. Computation of the SCS (dB) by the IE and CIE methods for an ellipsoidal scatterer.

We consider in Figure 7 a second test case: the scattering of a plane wave of incidence $(-1,0,0)$ and wavenumber $k_{2}=10$ by an ellipsoidal scatterer centered at the origin and with semi-axis $a=1, b=0.5$ and $c=0.3$ respectively along $\left(O x_{1}\right),\left(O x_{2}\right)$ and $\left(O x_{3}\right)$. The obstacle is characterized by the physical constants $\rho_{r}=1.1, c_{r}=1.2$ and $\gamma_{1}=5$. We consider that the reference IE solution is given for $N E=3000$ edges. As it can be observed, a good accuracy is obtained for the IE method with $N E=1080$ edges. The CIE method converges towards the reference solution a little bit more slowly than the IE method. This is due to the limit of validity of the local condition which penalizes the accuracy of the integral method and becomes prevailing. To precise the convergence of the method, we depict in Table 2 the quadratic relative error on the SCS according to the mesh refinement. Despite this lower convergence, we must always have in mind that the size of the linear system related to the CIE method is three times smaller than for the IE method. This finally makes the method competitive. 


\section{REFERENCES}

[1] X. Antoine, Conditions de Radiation sur le Bord. Ph.D. Thesis, No. d'ordre 395, Université de Pau et des Pays de l'Adour, France (1997).

[2] X. Antoine, Fast approximate computation of a time-harmonic scattered field using the on-surface radiation condition method. IMA J. Appl. Math. 66 (2001) 83.

[3] X. Antoine and H. Barucq, On the construction of approximate boundary conditions for solving the interior problem of the acoustic scattering transmission problem, in Domain Decomposition Methods in Science and Engineering. R. Kornhuber, R. Hoppe, J. Periaux, O. Pironneau, O. Widlund, J. Xu, Eds., Springer Series. Lect. Notes Comput. Sci. Engrg. 40 (2004) 133-140.

[4] X. Antoine, H. Barucq and L. Vernhet, Approximate solution for the scattering of a time-harmonic wave by a homogeneous dissipative obstacle. Internal Report MIP 00-20, Laboratoire MIP, Toulouse (2000).

[5] X. Antoine, H. Barucq and L. Vernhet, High-frequency asymptotic analysis of a dissipative transmission problem resulting in generalized impedance boundary conditions. Asymptot. Anal. 26 (2001) 257.

[6] X. Antoine, A. Bendali and M. Darbas, Analytic preconditioners for the electric field integral equation. Internat. J. Numer. Methods Engrg. 61 (2004) 1310-1331.

[7] X. Antoine, A. Bendali and M. Darbas, Analytic preconditioners for the boundary integral solution of the scattering of acoustic waves by open surfaces. J. Comput. Acoustics, Special Issue on High Performance Scientific Computing in Acoustics 13 (2005). To appear.

[8] A. Bendali, Approximation par éléments Finis de surface de problèmes de diffraction des ondes électromagnétiques. Thèse de Doctorat, Université Paris VI (1984).

[9] A. Bendali and M. Souilah, Consistency estimates for a double-layer potential and application to the numerical analysis of the boundary-element approximation of acoustic scattering by a penetrable object. Math. Comp. 62 (1994) 65.

[10] B. Carpinteri, I.S. Duff and L. Giraud, Experiments with sparse preconditioning of dense problems of electromagnetic applications. Technical Report TR/PA/00/04, CERFACS, France (2000).

[11] B. Carpinteri, I.S. Duff and L. Giraud, Sparse pattern selection strategies for robust Frobenius norm minimization preconditioners in electromagnetism. Numer. Linear Algebra Appl. 7 (2000) 667.

[12] J. Chazarain and A. Piriou, Introduction to the Theory of Linear Partial Differential Equations. North-Holland, Amsterdam/New-York (1982).

[13] K. Chen and P.J. Harris, Efficient preconditioners for iterative solution of the boundary element equations for the threedimensional Helmholtz equation. Appl. Numer. Math. 36 (2001) 475.

[14] S.H. Christiansen and J.C. Nédélec, Des préconditionneurs pour la résolution numérique des équations intégrales de frontière de l'acoustique. C. R. Acad. Sci. Paris Sér. I Math. 330 (2000) 617.

[15] P.G. Ciarlet, Handbook of Numerical Analysis, Vol. II, Finite Element Methods (Part I). P.G. Ciarlet and J.-L. Lions, Eds., Elsevier Science Publisher, North-Holland, Amsterdam (1991).

[16] D. Colton and R. Kress, Integral Equation Methods in Scattering Theory. Krieger Publishing Company (1992).

[17] M. Costabel, Boundary integral operators on Lipschitz domains: elementary results. SIAM J. Math. Anal. 19 (1988) 613.

[18] M. Costabel and E. Stephan, A direct boundary integral equation method for transmission problems. J. Math. Anal. Appl. 136 (1985) 367.

[19] E. Darrigrand, Coupling of fast multipole method and microlocal discretization for the 3-D Helmholtz equation. J. Comput. Phys. 181 (2002) 126.

[20] E. Darve, The fast multipole method. I. Error analysis and asymptotic complexity. SIAM J. Numer. Anal. 38 (2000) 98.

[21] E. Darve, The fast multipole method: numerical implementation. J. Comput. Phys. 160 (2000) 195.

[22] R. Djellouli, C. Farhat, A. Macedo and R. Tezaur, Three-dimensional finite element calculations in acoustic solution scattering using arbitrarily convex artificial boundaries. Internat. J. Numer. Methods Engrg. 53 (2002) 1461.

[23] D.S. Jones, An improved surface radiation condition. IMA J. Appl. Math. 48 (1992) 163.

[24] R.E. Kleinman and P.A. Martin, On single integral equations for the transmission problem of acoustics. SIAM J. Appl. Math. 48 (1988) 307.

[25] G.A. Kriegsmann, A. Taflove and K.R. Umashankar, A new formulation of electromagnetic wave scattering using the on-surface radiation condition approach. IEEE Trans. Antennas Prop. 35 (1987) 153

[26] D. Levadoux, Étude d'une équation intégrale adaptée à la résolution haute-fréquence de l'équation d'Helmholtz. Thèse de Doctorat, Université Paris VI (2001).

[27] D. Levadoux and B. Michielsen, Nouvelles formulations intégrales pour les problèmes de diffraction d'ondes. ESAIM: M2AN 38 (2004) $157-175$.

[28] J.C. Nédélec, Acoustic and Electromagnetic Equations, Integral Representations for Harmonic Problems. Springer-Verlag, New York. Appl. Math. Sci. 144 (2001).

[29] F. Rellich, Über das asymptotische verhalten der lösungen von $\Delta u+\lambda u=0$, in unendlichen gebieten, Jahresber. Deutch. Math. Verein 53 (1943) 57.

[30] V. Rokhlin, Rapid solution of integral equations of scattering theory in two dimensions. J. Comput. Phys. 86 (1990) 414. 
[31] S.M. Rytov, Calcul du skin-effect par la méthode des perturbations. J. Phys. USSR 2 (1940) 233.

[32] Y. Saad, Iterative Methods for Sparse Linear Systems. PWS Pub. Co., Boston (1996).

[33] T.B.A. Senior, Impedance boundary conditions for imperfectly conducting surface. Appl. Sci. Res. B. 8 (1960) 418.

[34] T.B.A. Senior, Approximate boundary conditions for homogeneous dielectric bodies. J. Electromagnet. Wave 9 (1995) 1227.

[35] T.B.A. Senior, Generalized boundary conditions for scalar fields. J. Acoust. Soc. Amer. 97 (1995) 3473.

[36] T.B.A. Senior and J.L. Volakis, Approximate Boundary Conditions in Electromagnetics. IEE Electromagnetic Waves, Serie 41, London (1995).

[37] T.B.A. Senior, J.L. Volakis and S.R. Legault, Higher order impedance and absorbing boundary conditions. IEEE Trans. Antennas Prop. 45 (1997) 107.

[38] O. Steinbach and W.L. Wendland, The construction of some efficient preconditioners in the boundary element method. Adv. Comput. Math. 9 (1998) 191.

[39] L. Vernhet, Approximation par éléments finis de frontière de problèmes de diffraction d'ondes avec condition d'impédance. Ph.D. Thesis, Université de Pau et des Pays de l'Adour, No. 400, France (1997).

[40] L. Vernhet, Boundary element solution of a scattering problem involving a generalized impedance boundary condition. Math. Methods Appl. Sci. 22 (1999) 587.

[41] D.S. Wang, Limits and validity of the impedance boundary condition on penetrable surfaces. IEEE. Trans. Antennas Prop. 35 (1987) 453.

To access this journal online:

www.edpsciences.org 